\title{
Dual-band X/Ku Reflectarray Antenna Using a Novel FSS-Backed Unit-Cell with Quasi- Spiral Phase Delay Line
}

\author{
Iman Derafshi, Nader Komjani, Ensieh Ghasemi-Mizuji and Mohammad Mohammadirad, \\ Department of Electrical Engineering, Iran University of Science and Technology, Tehran 1684613114, Iran, \\ e-mail:iman_darafshi@elec.iust.ac.ir,n_komjani@iust.ac.ir,ensiyeh.ghasemi@ee.iust.ac.ir, \\ mohammadirad@ee.iust.ac.ir
}

\begin{abstract}
A novel FSS-Backed reflectarray unit cell is introduced to design a dual-band X/Ku Reflectarray Antenna (RA). A Kuband RA based on this FSS-Backed cell element is designed and located on the top of a conventional X-band RA. Actually, the band-stop Frequency Selective Surface (FSS) property is applied to a wideband element to create isolation between $X$ and $K u$ band RAs which utilizing the same radiating element. A wideband cell element with attached quasi-spiral phase delay line is employed for phase compensation in both bands. As a FSS-backed cell element, the remarkable feature of the proposed dual band unit cell compared to previous works is its possibility of operation in closer frequency bands in comparison with other dual band FSS-backed RAs. Two reflectarrays with aperture size of $7.65 \lambda \times 7.65 \lambda$ and $7.35 \lambda \times 7.35 \lambda$ are designed, fabricated and measured for $X$ band and $\mathrm{Ku}$ band, respectively. Measured results show 1-dB gain bandwidth of $12 \%$ for $X$ band and $11 \%$ for $\mathrm{Ku}$ band which demonstrate wideband operation of this dual-band reflectarray antenna.
\end{abstract}

Index Terms - Delay line, dual band, FSS-Backed reflectarray antenna, quasi-spiral.

\section{INTRODUCTION}

Reflectarray antennas compared to other high gain antennas are used extensively in some long distance communication systems, due to their numerous advantages. Nowadays, these antennas are applicable for dual-band applications. If frequency bands are chosen close to each other, a single layer dual-band reflectarray antenna can be used [1]-[3]. Compared to multi-layer structures, this technique leads to reduction of the antenna's volume, but because of increasing mutual coupling between both the elements for different bands, the design would be difficult. To achieve operational bands considerably at a great distance, design in multi-layer configuration is preferred occasionally [4]-[6]. However, employing multi-layer configuration causes some restrictions in selecting thickness of substrates and the elements type as well as complexity of fabrication process.

In [7] a new type of reflectarray antennas (FSS-Backed reflectarray) for multi-band applications is introduced. Due to more degrees of freedom for design and good isolation between both band elements without any limitations in selection of thickness of substrates and type of elements, this type of antenna is preferred to the previous mentioned ones in some applications. Recently, some attempts have been done to employ these antennas for dual-band purposes [8]-[10]. In these structures, 
variable-size element technique is usually applied for compensation of spatially phase error of incoming wave. It is worth mentioning that by using the FSSs introduced in [7]-[9] designing dual band reflectarray in the frequency bands which are close to each other is not possible. In other words, obtaining reflection and transmission properties for two close wide bands would not be achievable with the elements introduced in the above literature.

In this paper, two RAs are designed and fabricated for dual band operation in $\mathrm{X}$ and $\mathrm{Ku}$ band. Both RAs are exploiting an element with attached phase delay lines as the main radiating element while a double layer FSS element is added beneath the Ku-band (17-19 GHz) element to obtain transmission property in X-band (8-9 GHz) and simultaneously simulating the ground plane in $\mathrm{Ku}$ band. By virtue of this structure, designing two stacked RA for closer frequency bands in comparison with the other dual band FSS-backed RAs will be possible which has not been previously reported. Thanks to the linear phase response of the delay line method [11]-[13] and it's lower tolerance in manufacturing. It has been used as the phase shifting mechanism for main element of the designed RAs in this work. As mentioned above a wideband element based on quasi-spiral phase delay line is utilized for designing a FSS-backed reflectarray. In fact, the FSS layer is considered as ground plane for the top Ku-band and also as a transparent layer for the bottom X-band RA.

\section{ANTENNA CONFIGURATION}

Fig. 1 shows the schematic view of the proposed dual band RA. As it is depicted in Fig. 1(a), the FSS-Backed reflectarray antenna for Ku band is placed on the top of X band conventional reflectarray by a $\left(\mathrm{h}_{\mathrm{i}}=1 \mathrm{~cm}\right)$ spacer. It is to be noted that all the dimensions for spacers were chosen based on achievable mechanical accuracy and material availability. The design has been carried out so that the center-fed $\left(\theta_{\mathrm{f}}^{\prime}=0^{\circ}, \phi_{\mathrm{f}}^{\prime}=0^{\circ}\right)$ FSS-backed Ku-band reflectarray antenna and $-15^{\circ}\left(\theta_{\mathrm{f}}=15^{\circ}, \phi_{\mathrm{f}}=180^{\circ}\right)$ offsetfed X-band RA produce $-15^{\circ}\left(\theta_{b}^{\prime}=15^{\circ}, \phi_{b}^{\prime}=180^{\circ}\right)$ and $15^{\circ}\left(\theta_{b}=15^{\circ}, \phi_{b}=0^{\circ}\right)$ off-broadside beams in elevation plane, respectively.

In Fig. 1(b) we can see the location of the Ku-band RA on top of the X-band RA and compare its size with the bottom RA. The size of this Ku-band FSS-Backed RA is about $1 / 4$ of the X-band one. According to Fig. 1(c), the FSS-backed RA is composed of two layers. The phasing elements are etched on the top surface of the upper layer and FSS elements are printed on both sides of the bottom layer, where the free space distance between these two layers $\left(\mathrm{d}_{\mathrm{ku}}\right)$ is $2 \mathrm{~mm}$. Also, as can be seen in Fig. 1(c) the X-band elements are etched on top of the bottom layer which is backed by a ground plane at distance of $2 \mathrm{~mm}\left(\mathrm{~d}_{\mathrm{x}}\right)$ to increase the bandwidth. All the used substrates in this design are RO-4003 with thickness 32 mil.

\section{A. Design of Ku band element}

The unit-cell configuration for Ku-band is shown in Fig. 2. As it can be seen from Fig. 2(a), this element consists of two RO-4003 substrates separated by a $2 \mathrm{~mm}$ spacer $\left(\mathrm{d}_{\mathrm{ku}}\right)$ from each other. The phasing element is a ring with attached quasi-spiral phase delay lines (Fig. 2(b)) which is etched on 
the top of the upper substrate [14]. The elements of Fig. 2(c) and Fig. 2(d) printed on both sides of the bottom substrate are used as FSS layer (Fig. 3(a)).The main effect of $2 \mathrm{~mm}$ spacer is to reduce the coupling between the elements on top (radiating) and bottom (FSS) layers and thus increasing the bandwidth of the RA [15]. Final dimension of the unit cell is $9 \mathrm{~mm} \times 9 \mathrm{~mm}$. The design process of the proposed cell element is originated from the method presented in [7] for multiband-multi polarization elements. As the starting point, the dimensions of the FSS layer are maintained while the length of the phase delay lines of the radiating element is changed to achieve a large phase variation range. Then, the FSS layer is adjusted to obtain maximum reflection in $\mathrm{Ku}$ band and at the same time minimum transmission in X-band.
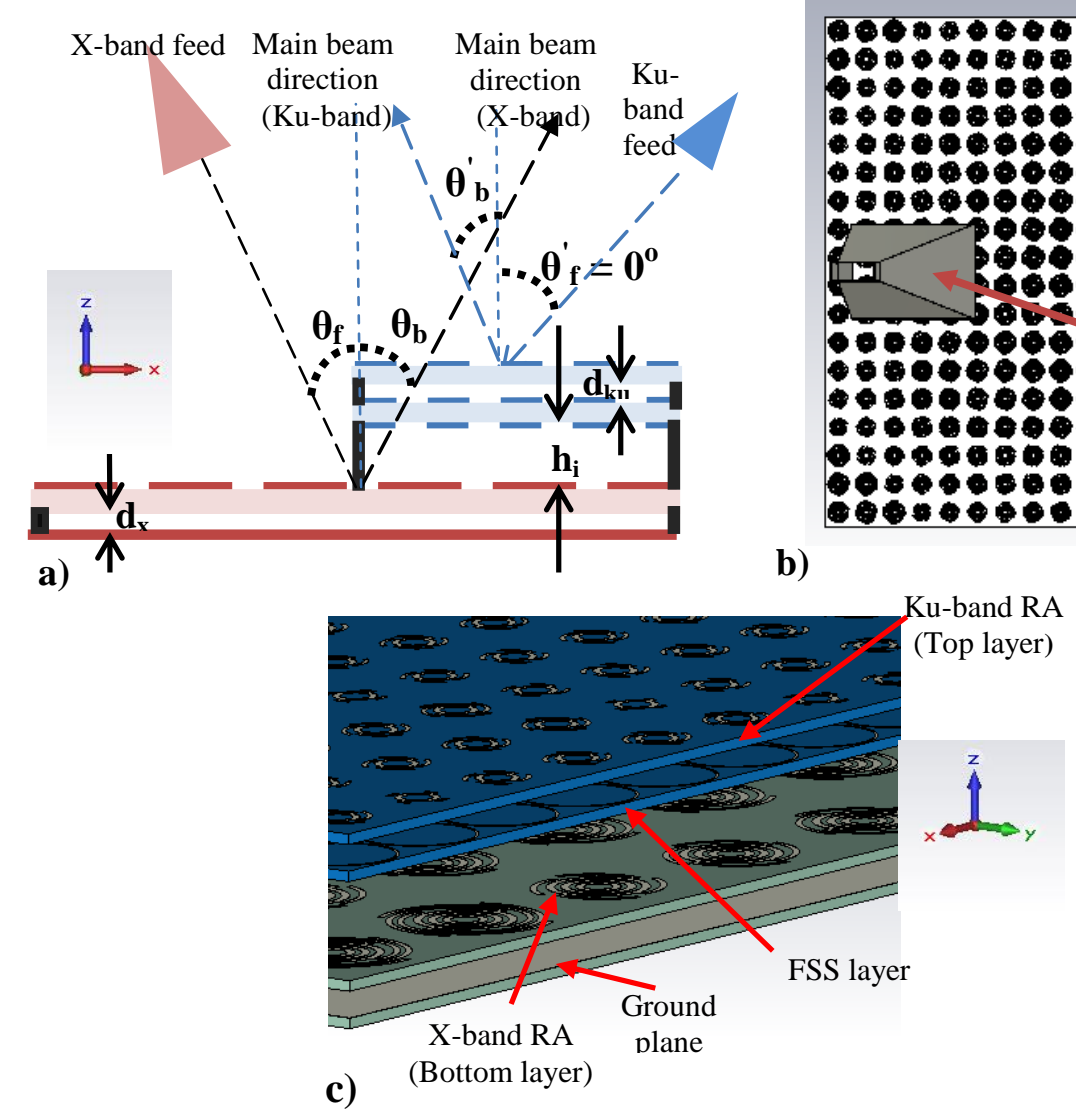

Fig. 1. a) Side view, b) top view and c) 3D view.

Based on the method in [7] two different FSS-backed unit cells are studied to get more insight on the cell element of Fig. 2. As shown in Fig. 3, first element consists of a phasing element on top layer and a ring element on bottom layer as the FSS-layer, for frequency range of $17.5 \mathrm{GHz}$. The plot of Fig. 3 illustrates the reflection coefficient for extreme values of $n$, where $n$ indicates the numbers of turn $\left(n=1\right.$ equals to a full turn $\left(360^{\circ}\right)$ ). The amplitude of reflection in $16.5-18.5 \mathrm{GHz}$ band is greater than $-1 \mathrm{~dB}$ (\%80 of reflection) which demonstrates that the FSS layer acts as a ground plane to some extent. On the other hand, the out of band performance of this element shows that: 1) most of the incident wave is transmitted at frequencies which are far enough from the aforementioned band. 2) 
Also, the magnitude of reflection coefficient for large values of $\mathrm{n}$ shows that about $25 \%$ of the incident wave will be reflected in the frequency range of $7.5-9 \mathrm{GHz}$ by this element. It is to be noted that to achieve a FSS-backed element where in the pass-band and stop-band occurs close to each other, it is necessary to decrease the amount of the latter reflection.

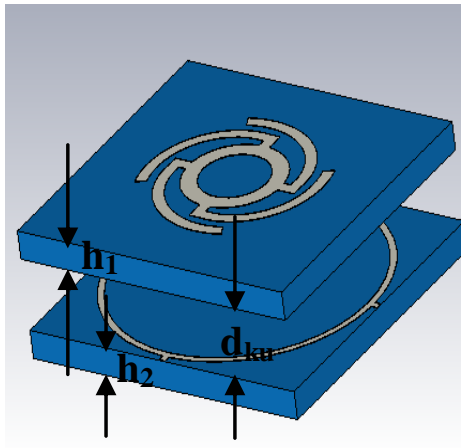

a)

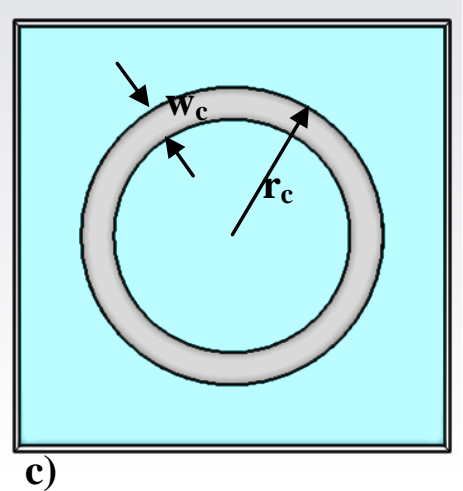

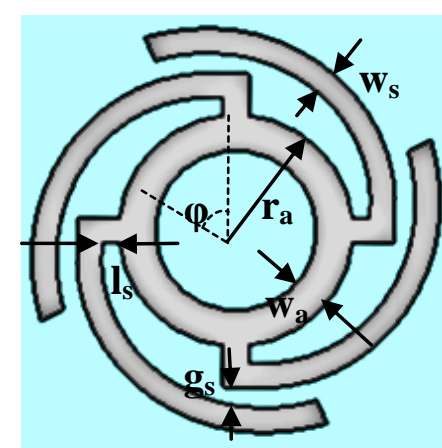

b)

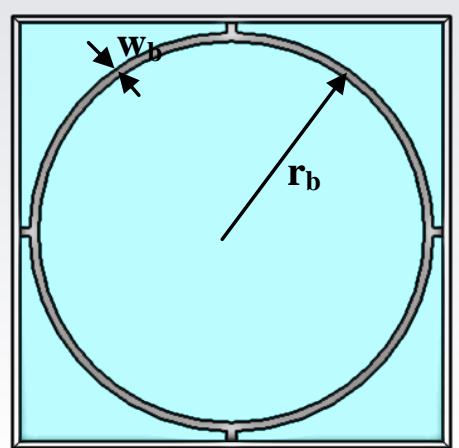

d)

Fig. 2. a) Schematic view of the proposed unit cell. b) Phasing element c) FSS element on bottom, d) FSS element on top.

Therefore, the element of Fig. 4 is used to obtain more reduction of the reflection coefficient in out of band, particularly at frequencies close to the design frequency. Similar to [16], the ring element of the FSS layer is connected to the adjacent elements by four stubs. According to [17], these additional stubs cause some reduction of the inductance in equivalent circuit of FSS element. On the other side, to maintain the resonance frequency, $\mathrm{f}_{\mathrm{r}}=\sqrt{L C}$, the capacitance in the equivalent circuit should be increased. Since the bandwidth of stop-band filters is proportional to $\sqrt{L / C}$, the stop band around 17.5 $\mathrm{GHz}$ will increase while the reflection coefficient out of the operational band (around $8 \mathrm{GHz}$ ) will decrease to $-7 \mathrm{~dB}$, as is shown in Fig. 4.

Based on the above investigation to achieve an appropriate performance in both bands, the FSS element in Fig. 5 is exploited which is a combination of both typical and modified elements in Fig. 3 and Fig. 4. The dimensions of the elements are optimized to attain proper operation in both bands (7.5-9 GHz and 17.2-18.2 GHz). The amplitude of the reflected wave for two different values of $n$ $\left(S_{11}\right)$ is shown in Fig. 5. As it is observed in this figure the amplitude of $S_{11}$ is less than $-9 d B$ in the frequency range of 7.5-9 GHz and in $\mathrm{Ku}$ band (17.2-18.2 GHz) $\mathrm{S}_{11}$ amplitude is more than $-0.8 \mathrm{~dB}$. Therefore, the goal is obtained. The final design parameters are as follow: $\mathrm{w}_{\mathrm{b}}=0.2 \mathrm{~mm}, \mathrm{r}_{\mathrm{b}}=4.3 \mathrm{~mm}$, 
$\mathrm{w}_{\mathrm{c}}=0.7 \mathrm{~mm}, \mathrm{r}_{\mathrm{c}}=3.2 \mathrm{~mm}$.

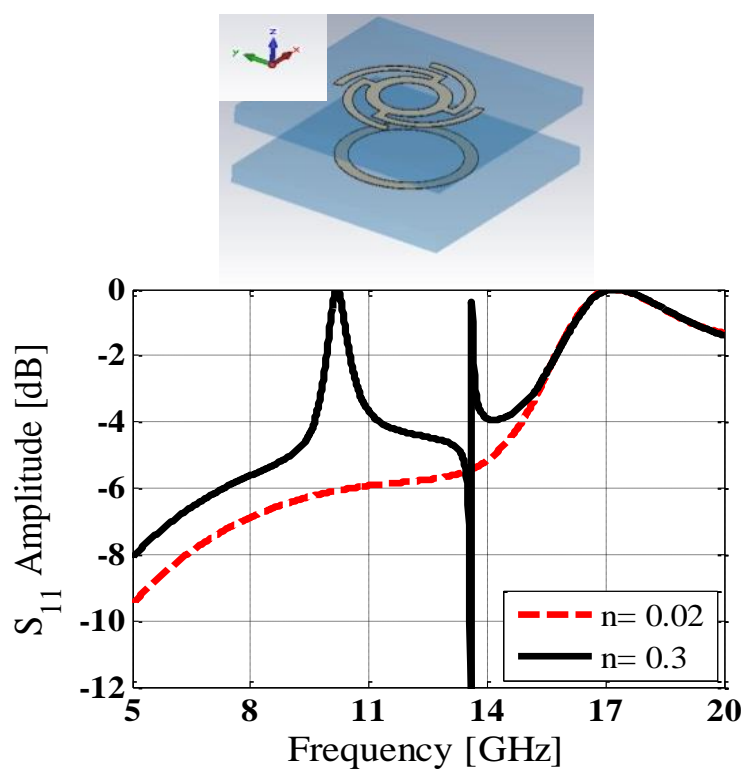

Fig. 3. FSS-backed element with a ring as FSS layer.
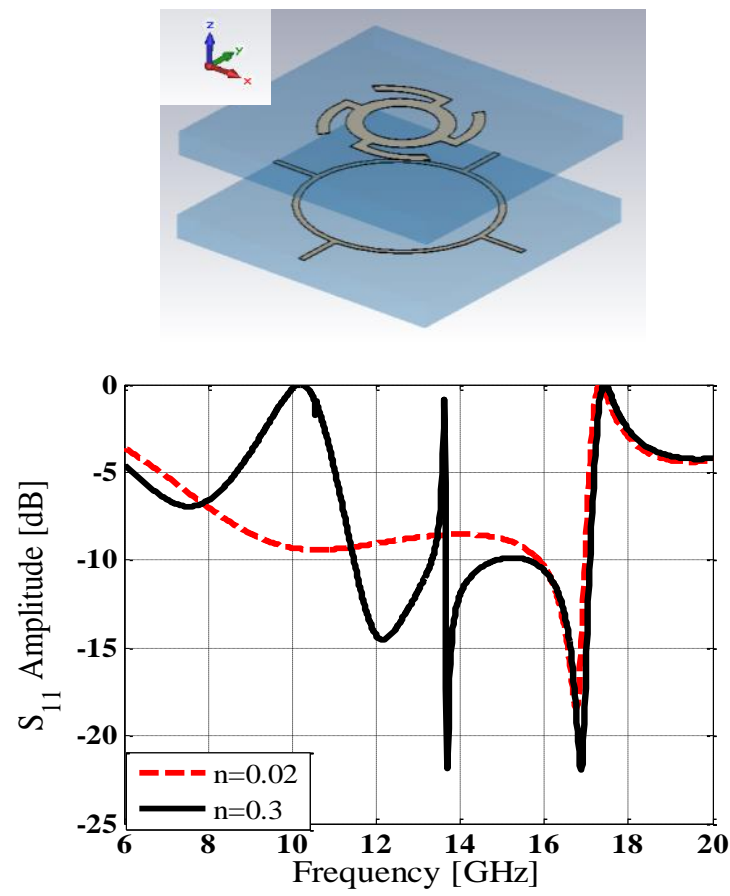

Fig. 4. FSS-backed element with a stub-loaded ring as FSS layer.

The phasing element of FSS-Backed RA (the upper layer in Fig. 2(a)) is a ring with four attached delay lines. Note that the phase shift is proportional to twice of the electric length of phase delay line [18]. Therefore, by varying the delay line length the necessary phase shift is attained. Note that the variable parameter of the phasing element is the turn value; therefore it is worthwhile to obtain the relation between the turn value and length of the delay line. The equation of the radius versus $\varphi$ for spiral line is expressed by (1): 


$$
r=r_{a}+l_{s}+(2 / \pi) \times\left(g_{s}+w_{s}\right) \times \varphi
$$

Where the parameters $\varphi, w_{s}, g_{s}, 1_{s}$ and $r_{a}$ are specified in Fig. 2(b). By substituting $\varphi=2 n \pi$ in (1):

$$
r=r_{a}+l_{s}+4 n\left(g_{s}+w_{s}\right)
$$

Where $\mathrm{n}$ is denoting the number of turn. The differential equation shown below can be considered:

$$
d l=r . d \phi=2 \pi r . d n
$$

By integrating and simplifying the formula, (4) is derived.

$$
l=2 n \pi\left[r_{a}+l_{s}+2 n\left(g_{s}+w_{s}\right)\right]
$$

The parameters $g_{s}$ and $w_{s}$ are optimized to obtain maximum matching with ring at $17.5 \mathrm{GHz}$ and the resonance dimensions of ring are designed in center frequency. The optimum parameters are obtained as: $\mathrm{w}_{\mathrm{s}}=0.35 \mathrm{~mm}, \mathrm{~g}_{\mathrm{s}}=0.2625 \mathrm{~mm}, 1_{\mathrm{s}}=0.2 \mathrm{~mm}, \mathrm{r}_{\mathrm{a}}=1.58 \mathrm{~mm}, \mathrm{w}_{\mathrm{a}}=0.5 \mathrm{~mm}$.
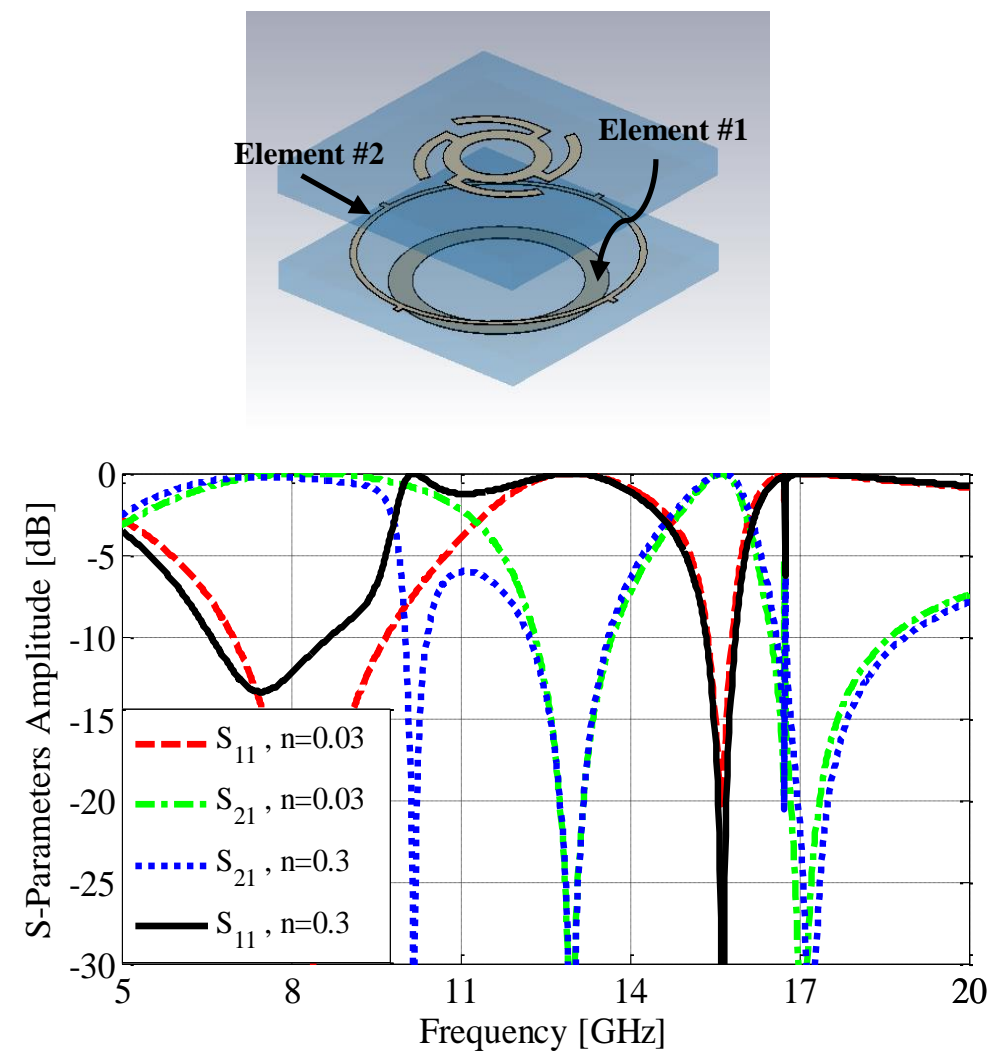

Fig. 5. The proposed FSS-backed reflectarray unit cell and its reflection response.

To check the operation of FSS for different values of phase delay lines length, the S-parameters are obtained using CST Microwave Studio. Fig. 6(a) shows the amplitude of $\mathrm{S}_{11}$ versus values of turn. As it is observed, at $17.5 \mathrm{GHz}$ the amplitude of reflection for a range of delay lines length is above $0.55 \mathrm{~dB}$. Also, the amplitude of reflected wave in Ku-band which is shown in Fig. 6(a) for several frequencies, demonstrates the ground plane role of FSS layer in Ku-band.

To get a better understanding, the performance of this unit cell in $\mathrm{X}$ band is examined. The $\mathrm{S}_{11}$ parameter versus different turn values of phase delay line is presented in Fig. 6(b). In the X band (8-9 
$\mathrm{GHz})$ and for various lengths of delay lines, $|\mathrm{S} 11|$ is under $-9 \mathrm{~dB}$ which means that the FSS-Backed RA transmits the incident wave completely in this frequency band.

The reflection phase of the FSS-backed cell element versus delay line length is depicted in Fig $.6(\mathrm{c})$. The phase response curves in Fig. 6(c) are parallel to each other for various frequencies which demonstrate wideband performance of this element. A phase shift range of $360^{\circ}$ for reflectarray is achieved for variation of $\mathrm{n}=0.02$ to 0.3 .

Since the Ku-band FSS-Backed RA occupies $1 / 4$ of the X band RA surface, our expectation would be a negligible variation of the transmission phase. The phase curves of Fig .6(c) demonstrate this transparency in X-band.
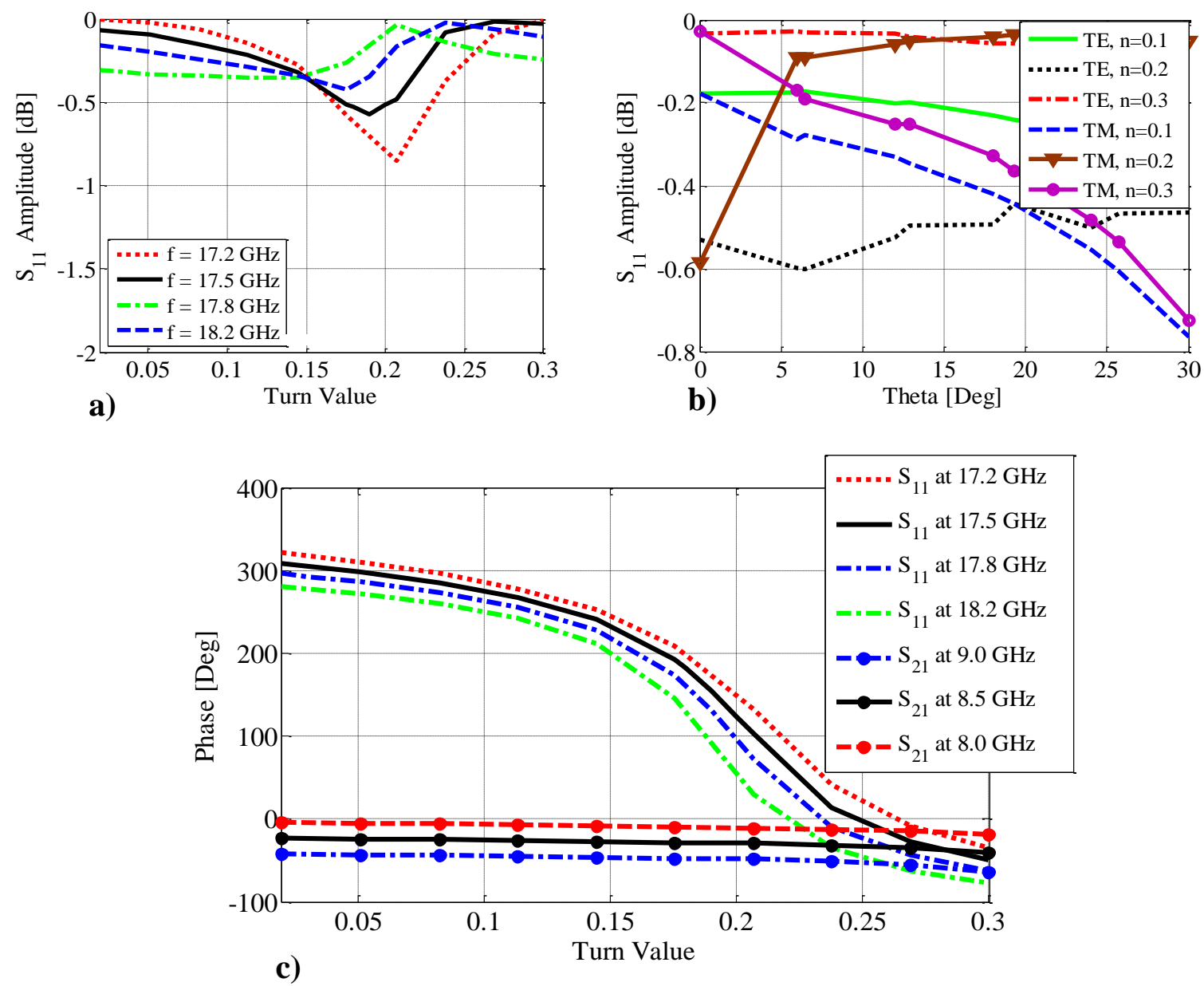

Fig. 6. $S_{11}$ amplitude versus turn value for (a) X-band and (b)Ku-band FSS-Backed unit cell. c) $S_{11}$ and $S_{21}$ phase versus values of turn for different frequencies.

Fig. 7(a) and. 7(b) show the effect of incidence angle (for Ku-band horn) on phase and amplitude response of the element in Fig. 5. It should be mentioned that the reflection coefficient is greater than $-0.8 \mathrm{~dB}$ for the maximum turn value and at the angles of incidence up to $\theta=30^{\circ}$. Furthermore, the magnitude of transmission coefficient for various turn values and TE/TM waves at different angles of incidence (for X-band horn) demonstrate the low sensitivity of the proposed unit-cell to position Xband feed. 

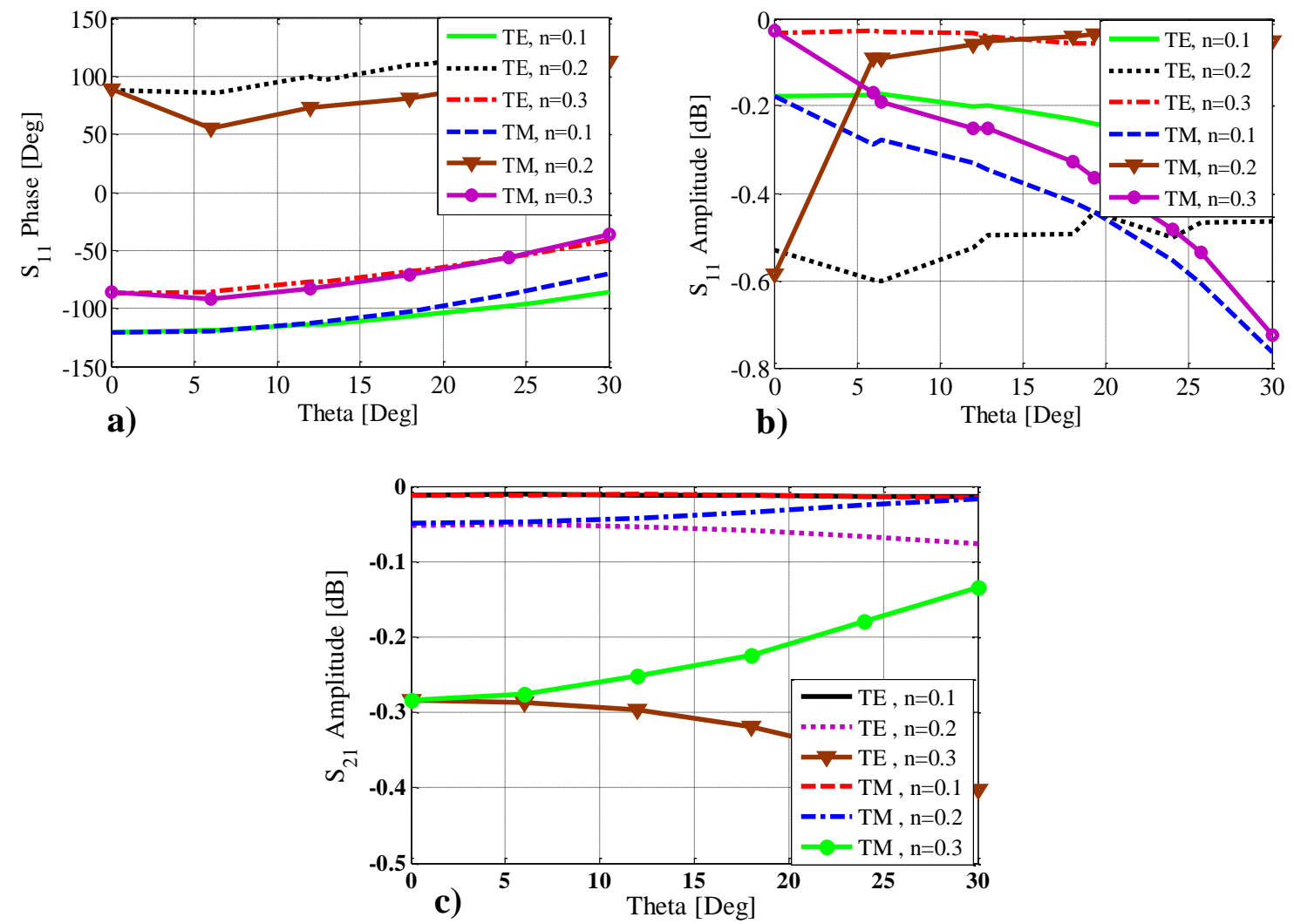

Fig. 7. $\mathrm{S}_{11}$ a) phase versus incident wave angle at 17.5.GHz. b) amplitude versus incident wave angle at 17.5.GHz. c) $\mathrm{S}_{21}$ amplitude versus incident wave angle at $8 \mathrm{GHz}$.

\section{B. Design of Ku band reflectarray}

The back-scattered field of the whole reflectarray in an arbitrary direction (u) is expressed as:

$$
E(\widehat{u})=\sum_{p=1}^{P} \sum_{q=1}^{Q} F\left(\vec{r}_{p q} \cdot \vec{r}_{f}\right) A\left(\vec{r}_{p q} \cdot \hat{u}_{0}\right) A\left(\widehat{u} \cdot \widehat{u}_{0}\right) \cdot \exp \left(-j k_{0}\left[\left|\vec{r}_{p q}-\vec{r}_{f}\right|+\vec{r}_{p q} \cdot \widehat{u}\right]+j \phi_{p q}\right)
$$

Where $\mathrm{F}$ and $\mathrm{A}$ are feed antenna pattern and unit cell element pattern, respectively. $\mathrm{r}_{\mathrm{pq}}$ and $\mathrm{r}_{\mathrm{f}}$ denote the position vector to $\mathrm{pq}_{\mathrm{th}}$ element and the position of the feed antenna, respectively. Also, $\mathrm{u}_{0}$ is the chosen main beam direction and $\varphi_{\mathrm{pq}}$ is the required phase shift at $\mathrm{pq}_{\mathrm{th}}$ element which is given as:

$$
\varphi_{p q}=k_{0}\left[d_{p q}-\left(x_{p q} \cos \varphi_{b}+y_{p q} \sin \varphi_{b}\right) \sin \theta_{b}\right]
$$

Where $\mathrm{k}_{0}$ is the free space wave number. And $\left(\mathrm{x}_{\mathrm{pq}}, \mathrm{y}_{\mathrm{pq}}\right)$ is the coordinate of $\mathrm{pq}_{\mathrm{th}}$ element and $\left(\theta_{\mathrm{b}}, \varphi_{\mathrm{b}}\right)$ is the main beam direction [18].

A $14 \times 14$ element center fed FSS-Backed reflectarray is designed and fabricated using cell element of Fig. 2. The reflectarray aperture is illuminated by a horn antenna with $F / D=1.38$. The RA was designed to produce a main beam at $\left(15^{\circ}, 180^{\circ}\right)$.

The required phase shift distribution on the surface of Ku band RA is depicted in Fig. 8. The delay line length for producing the phase shift in $\mathrm{Ku}$ band has been calculated from Fig. 6(c).

The fabricated RA antenna is shown in Fig. 9. As aforementioned, this antenna consists of two layers with distance of $2 \mathrm{~mm}$ from each other (Fig. 9(d)). The top view of the upper layer, the top 
view of the bottom layer and the back view of the bottom layer of Ku band RA are demonstrated in Fig. 9(a), 9(b) and 9(c), respectively.

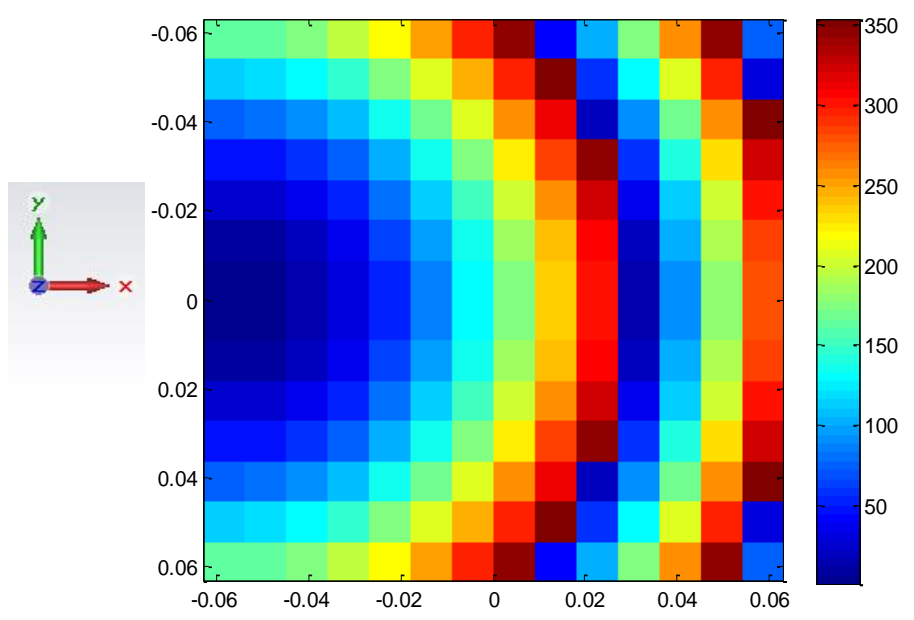

Fig. 8.The required phase shift for each element of FSS-Backed RA (Ku-band).

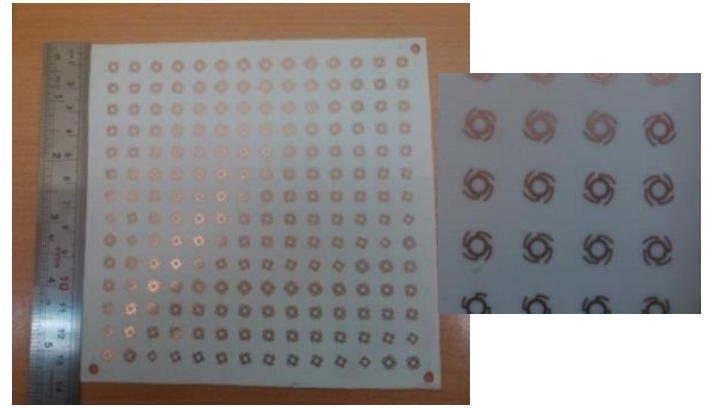

a)

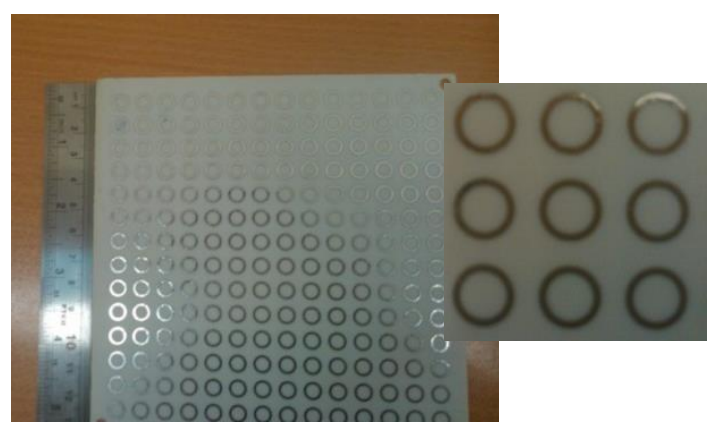

c)

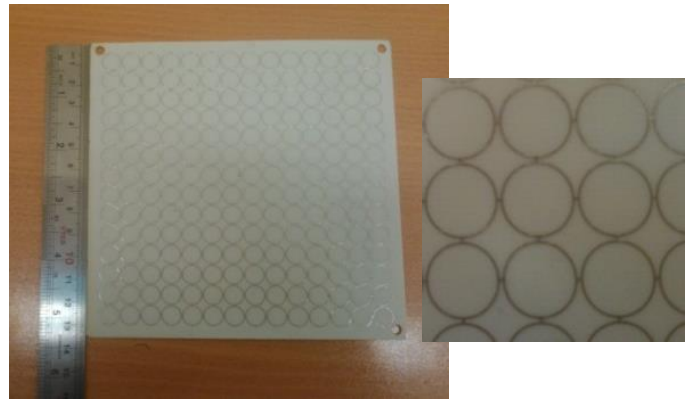

b)

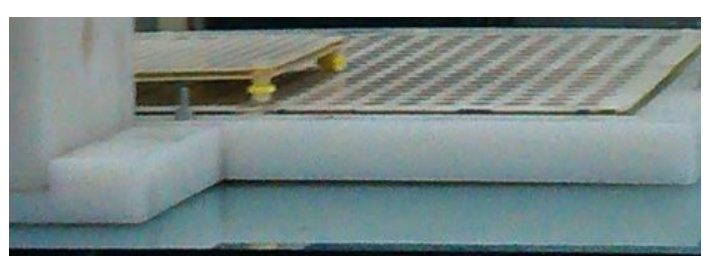

d)

Fig. 9. a) top view of FSS-Backed RA, b) top view of FSS layer, c) back view of FSS layer and d) side view of FSS-Backed RA where placed on top of X-band RA.

The measurement setup is shown in Fig. 10. The measured radiation pattern at $17.5 \mathrm{GHz}$ and the gain curve are represented in Fig. 11. As it is observed the main beam direction in E-plane is -15 deg off-broadside and the measured gain is $23.9 \mathrm{dBi}$ (aperture efficiency is $36 \%$ ) at $17.5 \mathrm{GHz}$. The measured 3-dB beam width and side lobe level are $6^{\circ}$ and $-15 \mathrm{~dB}$, respectively. Fig. 11(b) illustrates the measured gain versus frequency for the frequency range of $16-19 \mathrm{GHz}$, where the frequency range of $16.5-18.5 \mathrm{GHz}$ is measured as $1-\mathrm{dB}$ gain bandwidth. It can be seen that the peak gain occurs at a lower frequency with regard to design frequency, which can be due to fabrication tolerances. 


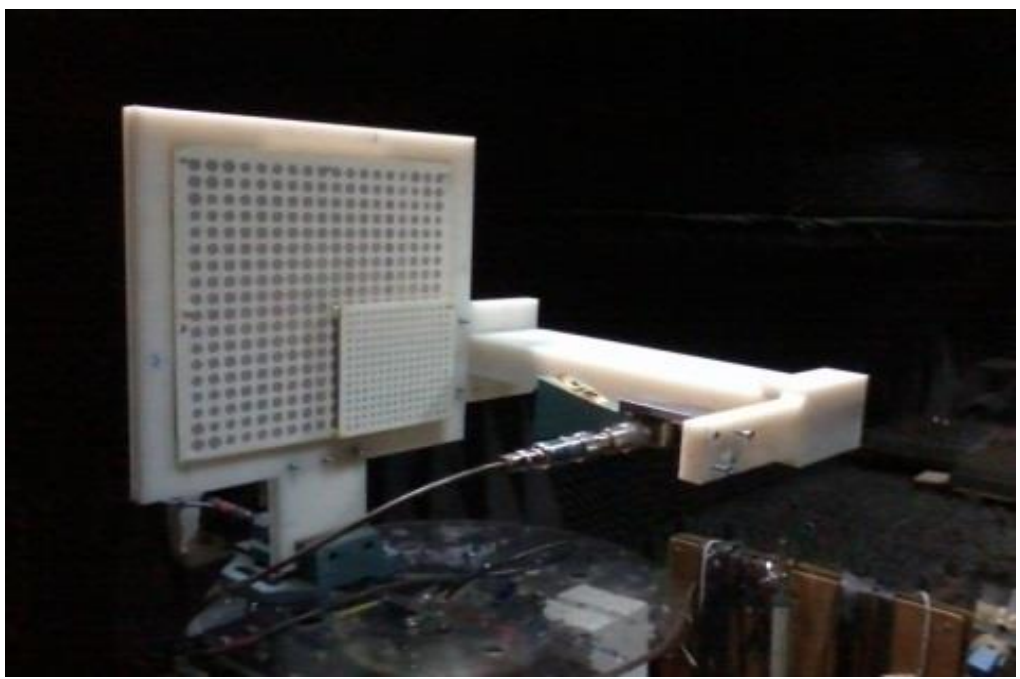

Fig. 10. The test setup of the mentioned FSS-Backed RA.

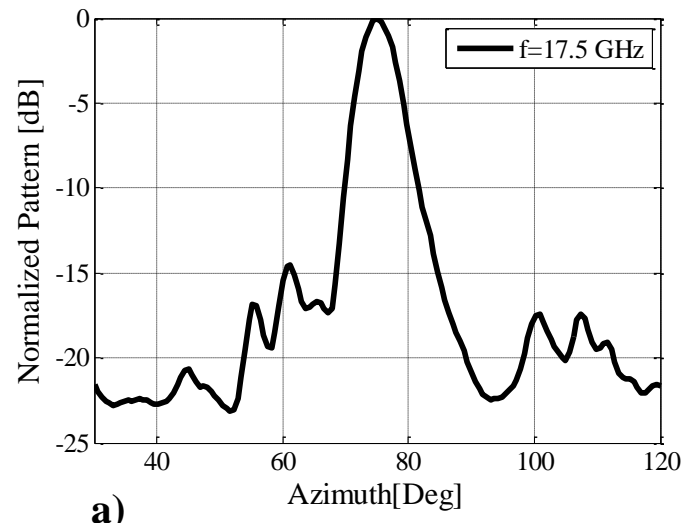

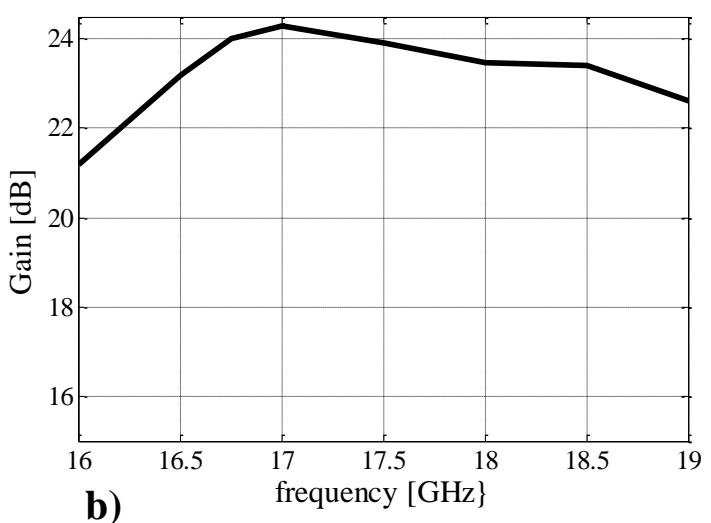

b)

Fig. 11 .a) Normalized pattern of FSS-Backed RA at 17.5 GHz. b) Gain versus frequency FSS-Backed RA.

\section{$C$. The effect of FSS-backed RA on the performance of the X band RA}

Eventually, the performance of the X-band RA is assessed in the presence of Ku band FSS-backed RA. The unit cell of X-band RA is shown in Fig. 12. This wideband element has been previously introduced by the authors [14], and to avoid redundancy is not repeated here.

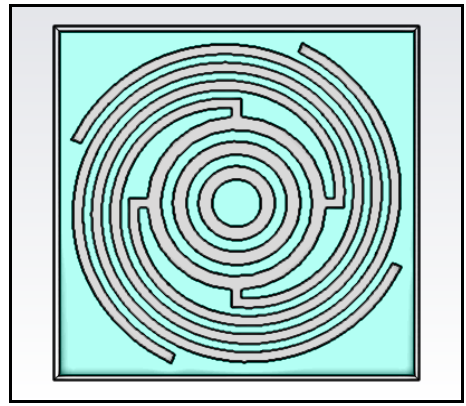

a)

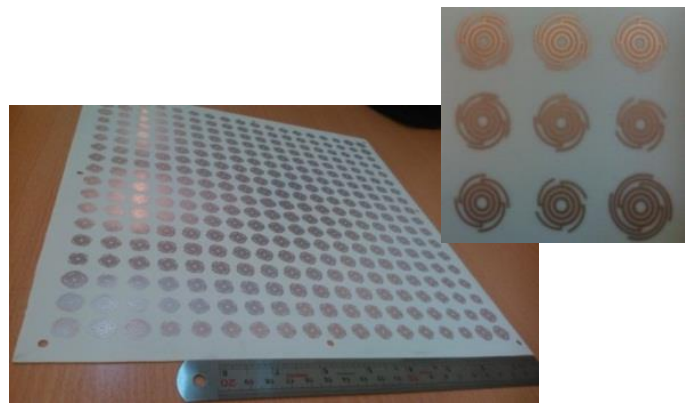

b)

Fig. 12. a) Unit cell element of X band RA and b) Prototype of fabriceted X band RA.

Based on [9] the larger spacing between two arrays results in smaller coupling. However, because of some restrictions on facilities this space was chosen to be $1 \mathrm{~cm}$. Utilizing the cell element in [14], Fig. 12(a), a 18×18 element reflectarray was designed and fabricated for X-band, Fig. 12(b), to 
produce a main beam in direction of $\left(15^{\circ}, 0^{\circ}\right)$. It should be noted that a $-15 \mathrm{deg}$ offset feed horn was used to illuminate this RA with $\mathrm{F} / \mathrm{D}=1.21$.

As it was shown in Fig. 7(c) the performance of FSS-backed RA has low sensitivity to the X-band horn and its angle of illumination. Therefore, it is expected that the location of FSS-backed RA on top of the X-band RA has negligible effect on performance of the X-band RA. However, to achieve more mechanical strength it was preferred to place it at the corner, as shown in Fig. 13(a).

The radiation pattern at $8.5 \mathrm{GHz}$ and gain curve vs. frequency for X-band RA are presented in Fig. 13(b) and .13(c). As it can be seen these results are given with and without Ku-band RA. Separately measurement of X-band RA shows the efficiency of the single X-band RA at $8 \mathrm{GHz}$ is about $63 \%$ ( $26.12 \mathrm{~dB}$ gain) while it is reduced to $59 \%(-26.4 \mathrm{~dB}$ gain) at the frequency of $8.5 \mathrm{GHz}$. However, when $\mathrm{Ku}$-band RA is located on the top of the $\mathrm{X}$ band $\mathrm{RA}$, the maximum gain at $8 \mathrm{GHz}$ is reduced to $24.68 \mathrm{~dB}$ ( $44 \%$ efficiency) and $24.75 \mathrm{~dB}$ gain ( $40 \%$ efficiency) at $8.5 \mathrm{GHz}$. Therefore, we can conclude an insertion loss of about $1.5 \mathrm{~dB}$ and $1.65 \mathrm{~dB}$ for the frequencies of $8 \mathrm{GHz}$ and $8.5 \mathrm{GHz}$ respectively. Also, According to this figure, the insertion loss is increased as the frequency increases. The 1-dB gain bandwidth for $\mathrm{X}$ band RA with $\mathrm{Ku}$ band RA is about $12 \%$.

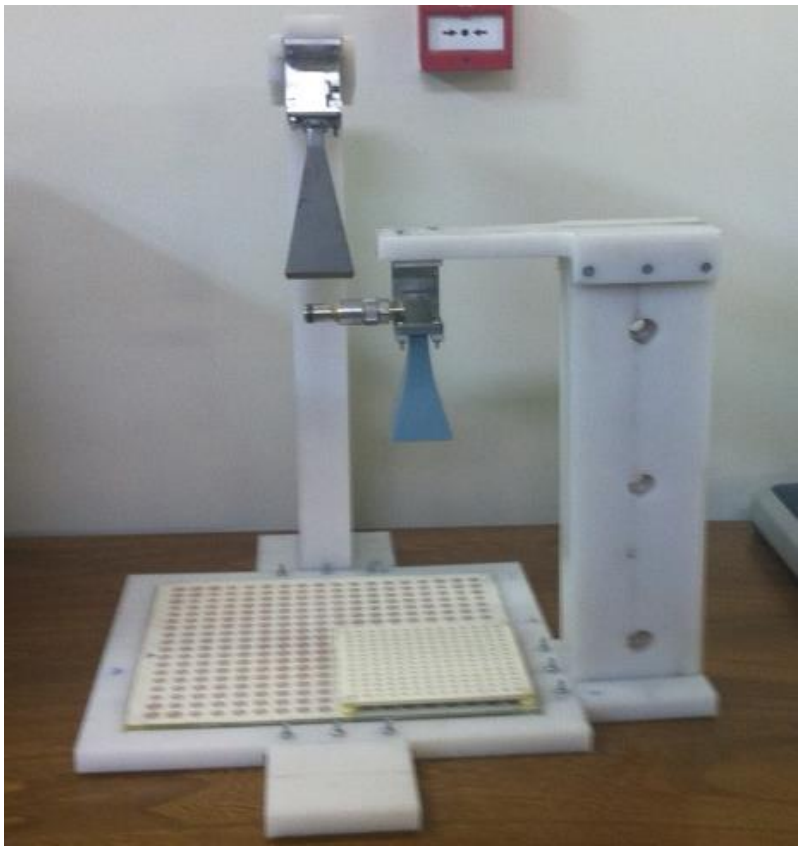

a)
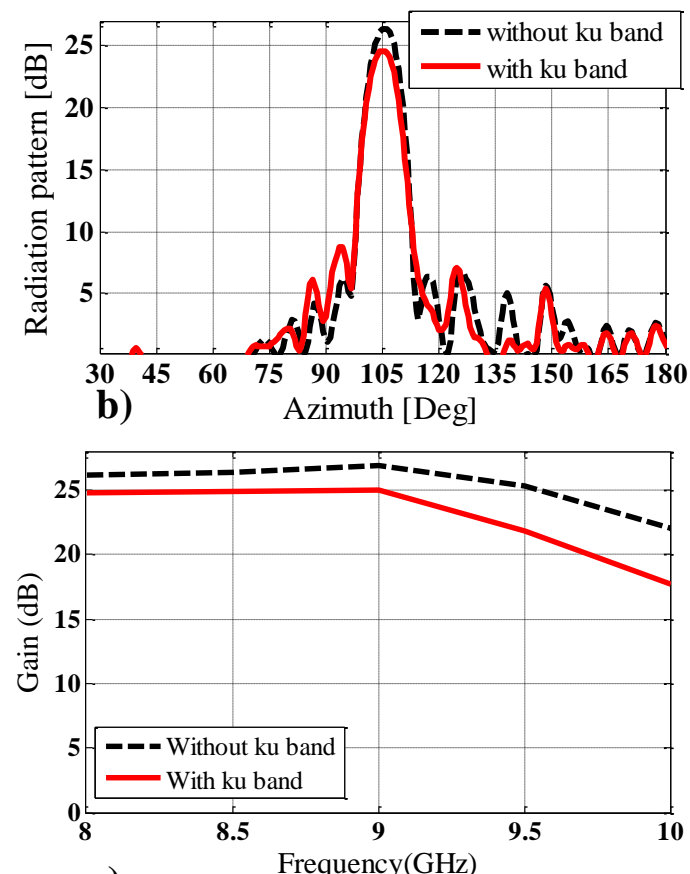

c)

Fig.13.a) Prototype of fabricated dual band RA, b) Gain versus frequency for X band RA with and without Ku-band FSSBacked RA and c) Radiation pattern X band RA with and without Ku band FSS-Backed RA.

\section{CONCLUSION}

In this study a $(\mathrm{X} / \mathrm{Ku})$ dual band reflectarray antenna was fabricated and measured. A new element consisted of a ring with four quasi-spiral delay lines was utilized as the unit cell element. Moreover, two FSSs were used to isolate the two bands. The FSS insertion loss is about $1.6 \mathrm{~dB}$ in $\mathrm{X}$ band. The measurements show the proper operation in both frequency bands. In addition, the efficiency in $\mathrm{X}$ band and $\mathrm{Ku}$ band are $40 \%$ and $36 \%$, respectively. The 1-dB gain bandwidth for $\mathrm{X}$ band in presence 
Journal of Microwaves, Optoelectronics and Electromagnetic Applications, Vol. 15, No. 3, September 2016236 DOI: http://dx.doi.org/10.1590/2179-10742016v15i3582

of $\mathrm{Ku}$ band is about $12 \%$ and for $\mathrm{Ku}$ band is $11 \%$.

\section{REFERENCES}

[1] M. R. Chaharmir, J. Shaker, N. Gagnon, and D. Lee, "Design of broadband, single layer dual-band large reflectarray using multi open loop elements,” IEEE Trans. Antenna Propag.,vol. 58, no. 9, pp. 2875-2883, Sep. 2010

[2] M. R. Chaharmir, J. Shaker, and N. Gagnon, "Broadband dual-band linear orthogonal polarization reflectarray," Electron. Lett.,vol. 45, no. 1, Jan. 2009.

[3] H. Hasani, and C. Piexeiro, "Dual-band, dual-polarized microstrip reflectarray antenna in Ku band," Loughborough Antennas \& Propagation Conference. Nov. 2012.

[4] C. Han, J. Huang, and K. Chang, "A high efficiency offset-fed X/Ka-dual-band reflectarray using thin membranes," IEEE Trans. Antenna Propag.,vol. 53, no. 9, pp. 2792-2798, Sep. 2005.

[5] C. Han, Ch. Rodenbeck, J. Huang, and K. Chang, "A C/Ka dual frequency dual-layer circularly polarized antenna with microstrip ring elements," IEEE Trans. Antenna Propag., vol. 52, no. 11, pp. 2871-2876, Nov. 2004.

[6] M. R. Chaharmir, J. Shaker, and M. Cuhaci, "Development of dual-band circularly polarized reflectarray," IEE Proceeding-Microwaves Antenna and Propagation, vol. 153, no. 1, pp. 49-54, Feb. 2006.

[7] J. Shaker, and M. Cuhaci, "Multi-band, multi-polarisation reflector-reflectarray antenna with simplified feed system and mutually independent radiation patterns," IEE Proceeding-Microwaves Antenna and Propagation, vol. 56, no. 12, pp. 3700-3706, Dec. 2008.

[8] M. R. Chaharmir, J. Shaker, and H. Legay, "Dual-band Ka/X reflectarray with broadband loop elements," IET Microw. Antenna Propag.,vol. 4, 1ss. 2, pp. 225-231, 2010.

[9] L. Li, Q. Chen, Q. Yuan, k. Sawaya, T. Maruyama, T.Furuno and Sh. Uebayashi, "Frequency selective reflectarray using crossed-dipole elements with square loops for wireless communication applications," IEEE Trans. Antenna Propag.,vol. 59, no. 1, pp. 89-99, Jan. 2011.

[10] Y. Chen, L. Chen, H. Wang, X. Gu and X. Shi, "Dual-band crossed-dipole reflectarray with dual-Band frequency selective surface,” IEEE Antenna Wireless Propag .Lett, vol. 12, pp. 1157-1160, 2013.

[11] R. D. Javor, X. Wu, and K. Chang, "Design and performance of a microstrip reflectarray antenna," IEEE Trans. Antenna Propag., vol. 43, no. 9, pp. 932-939, Sep. 1995.

[12] H. Hasani, M. Kamyab, and A. Mirkamali, "Broadband reflectarray antenna incorporating disk elements with attached phase-delay line," IEEE Antenna Wireless Propag. Lett, vol. 9, pp. 156-158, 2010.

[13] R. Sh. Malfajani, and Z. Atlasbaf, "Design and implementation of a broadband single layer circularly polarized reflectarray antenna," IEEE Antenna Wireless Propag.Lett, vol. 11, pp. 973-976, 2012.

[14] I .Derafshi, N. Komjani and M. Mohammadirad, "A Single Layer Broadband Reflectarray Antenna by Using Quasispiral Phase Delay Line,” IEEE AWPL,vol. 14, pp. 84-87, 2014.

[15] M. Mohammadirad, N. Komjani, M. Chaharmir, J. Shaker, A. Sebak, "Phase Error Analysis of the Effect of Feed Movement on Bandwidth Performance of a Broadband X-Ku Band Reflectarray," International Journal of RF and Microwave Computer-Aided Engineering/Vol. 23, No. 5, pp.17-526, 2012.

[16] J. Li, Q. Chen, Q. Yuan, and K. Sawaya, "FSS sandwiched reflectarray for dual-frequency application,"Microwave Conference Proceedings (APMC), Asia-Paciflic. pp. 877 - 880, 2010.

[17] B. Munk, Finite antenna arrays and FSS. Hoboken, N.J.: IEEE Press/Wiley-Interscience, 2003.

[18] J. Huang and J. A. Encinar, Reflectarray Antennas. New York: IEEE Press, Wiley-Interscience, 2008. 